\title{
The relation of field dependence to signal detection while imaging
}

\author{
FRANCIS J. DI VESTA \\ Pennsylvania State University, University Park, Pennsylvania 16802 \\ and \\ ANGELA BARTOLI \\ Shippensburg State College, Shippensburg, Pennsylvania 17257
}

\begin{abstract}
Twelve field-dependent (FD) and 12 field-independent (FI) males were administered 260 trials of a signal detection task in four phases. Signal type (auditory, visual, or no signal) was crossed with four sets of imaging treatments (pure visual representations, pure sounds without visual counterparts, and two sets representing sounds with visual counterparts). Detection of a signal in isolation was $90 \%$ accurate, as was the detection of no signal while imaging. Cross-modality imaging interfered with detection of the stimulus but to a significantly lesser extent than did same-modality imaging. In all detection/imaging treatments, FIs were significantly more sensitive to the signal than were FDs; however, FDs and FIs did not differ in accuracy of detecting isolated signals. The results are discussed in terms of the commonalities shared by perception and imaging as they appear in the Perky effect and cognitive style.
\end{abstract}

Several investigators (Brooks, 1968; Hebb, 1968; Segal \& Fusella, 1971) have suggested that imaging in a given modality engages some of the same mental machinery as perceiving in that modality, thereby occupying the same neurophysiological pathways (Broadbent, 1958). A similar view is suggested by Paivio (1971), who states that "strong evidence that visual perception and visual imagery tie-up one system and speech perception and verbal thinking another has been presented in a series of experiments by $L$. R. Brooks"' (1967, p. 144). Most often, the effects of perceptual processing dominate those of imagery. However, given deliberate conscious imaging, very weak external stimulation may go unnoticed. Thus, when external stimuli are at threshold, the percept and image may become functionally indistinguishable or ambiguous. In the present study, it is assumed that this effect is influenced by processing strategies or cognitive styles. Of concern is the cognitive style defined by the bipolar anchors of field dependence vs. field independence (FD/FI).

Differences along the FD/FI continuum relate to degree of differentiation of psychological functioning in perception (Witkin, Dyk, Faterson, Goodenough, \& Karp, 1974). Field-independent persons (FIs) use an analytical strategy whereby they attend to relevant cues and extract them from the accompanying "field" of stimuli. Thus, they are capable of restructuring a complex pattern in order to identify a simple figure within it. Field-dependent persons (FDs) are characterized by a global (holistic) strategy tending to be influenced by the strong gestalt of a larger pattern which deters identification of the target. For such persons, the relevant focal parts of the field are less separable from the surrounding field leading to functional ambiguity. Accordingly, the focal figure may remain undetected.

The FD/FI cognitive style was assumed to influence the Perky (1910) effect, since a global or analytic processing style appeared to be related to the separation of a percept and an image. Thus, in the "Perky effect," the external signal presented at threshold is assumed to be equal in intensity to an imaged representation (Segal \& Fusella, 1970), leading to an ambiguity. The detection of the signal is difficult presumably because it occupies the same processing channels as the image. A global, or holistic, strategy is a further potential source of difficulty in separating the signal and image. Because perceptual definition tends to be influenced by both external and internal stimuli and because the same system may be employed for processing both image and percept in a given modality, the FD will experience more difficulty in separating the two when experienced in the same modality. The FI being less constrained by extraneous stimuli should separate the two facets of experience more easily than the FD.

If we obtain an effect of FI/FD on the strength of the Perky effect, we would have to consider whether it could be entirely accounted for by differences in imaging ability. There are three reasons why we believe that differences in FI/FD are not entirely subsumed by differences in imaging ability: (1) In a previous study (Di Vesta, Ingersoll, \& Sunshine, 1971), 
spatial relations and embedded figures tests and two imagery (vividness and control of imagery) tests were administered to a sample of subjects from the same population as the one used in the present study. Correlations between the scores of each imagery test and each spatial relations test were calculated. Control of imagery and performance on embedded figures tests were found to comprise orthogonal factors. (2) The difference between FDs and FIs appears to be in the way they account for their images rather than in their abilities to image. The FD is more likely to attribute imaged events to an external, rather than an internal, source (Goldberger \& Holt, 1958; Segal, 1972). In a perceptual isolation experiment, Silverman, Cohen, Shmavonian, and Greenberg (1961) showed that FDs had difficulty in discriminating and in integrating sensory information. Their evidence indicated that FIs evaluated both internal and external cues more realistically than did with the FDs. (3) Segal, in a personal communication to Richardson (1969) also suggested that individual differences in reliance on external or internal cues in identifying perceptual or imaginal sources of information would be greatest in ambiguous situations. For these three reasons, we surmised that FDs and FIs employed different strategies in detecting signals while imaging.

In addition to the above theoretical considerations, we were concerned with a methodological one. A pilot study had shown that sensitivity to an auditory signal while imaging another auditory signal was greater than sensitivity to a visual signal while imaging another visual signal. Upon examining this procedure and the data further, it appeared that this difference might have been due to the kind of stimuli used for imaging in the auditory conditions. When identifying the presence or absence of a signal, subjects had been requested to image, for example, "a telephone ringing," following the procedure employed by Segal and Fusella (1970). Such instructions tend to be ambiguous; some of our subjects reported producing a visual image (e.g., of a telephone), and others reported producing an auditory image (e.g., of the ringing sound) or a combined auditory and visual image (e.g., a telephone vibrating with a ringing sound). Accordingly, the present study was designed to include (1) a "new" auditory imaging condition in which subjects were instructed to image nonambiguous sound stimuli (e.g., imagine a crackling sound), including the "sound" portion of some of those previously incorporated in the "old" auditory/visual stimuli used in earlier studies (Segal \& Fusella, 1970); and, (2) an additional (new) visual imaging condition in which subjects were to image the visual stimuli contained within the old auditory/visual stimuli (e.g., image a telephone).

In summary, based on the assumption that imagery and perception in a given modality produce ambiguity because the same processing systems are em- ployed, it was hypothesized that an imaging task would inhibit performance on a signal detection task in which signal and image were both in the same modality, but would have a lesser effect on crossmodality tasks in which the image and signal were experienced in different modalities. A second hypothesis was that FI subjects would be less susceptible to the incidental features produced by imaging and thus would be more able to detect the externally presented signal when it was in the same modality. A third hypothesis touched upon an issue regarding how best to explore these hypotheses. Specifically, this hypothesis was that the effect of an uncontaminated auditory image on the detection of an auditory signal would be equivalent to the effect of an uncontaminated visual image on the detection of a visual signal. Conversely, a mixed (visual and auditory) event, since it has the potential of evoking either an auditory or visual image, would have a less adverse effect on detection of the signal.

\section{METHOD}

The experiment was conducted in two sessions with the same four phases in each: practice, preimaging (signal detection without imaging) test trials, signal detection while imaging test trials, and postimaging (signal detection without imaging) test trials. The subjects were males classified as either field-dependent (FD) or field-independent (FI). The task was detecting the presence or absence of an externally presented tone (auditory signal) or figure (visual signal). When either signal was presented, it was at low intensity. The image accompanying a signal was from a class of visual or auditory events based on earlier studies (old) or on visual or auditory events devised for the present study (new). These four sets of materials are described in detail below. The main part of the experiment (the signal detection while imaging task) involved a $2 \times 3 \times 4$ design with cognitive style (FD/FI) as the between-subjects variable and type of signal (visual, auditory, and none) and class of imagined events (see Imaging Tasks below) as the within-subjects variables.

\section{Subjects}

The subjects were 24 male undergraduate students enrolled in an introductory educational psychology course. These subjects had been selected from a pool of 150 male students who had been administered the Hidden Figures Test described below. Credit toward the final grade was awarded to the subjects upon voluntary participation in the experiment. None of the subjects had participated previously in an experiment with similar requirements.

\section{Cognitive Style Test}

Cognitive style was measured by the Hidden Figures Test, Form CF-1, administered according to the manual of instructions (Witkin, Oltman, Raskin, \& Karp, 1971). The test, which measures field dependence (FD) and field independence (FI), consists of a fivepage booklet. Following the general instructions and a sample test item, the body of the test contains 32 matching tasks. Each task requires the respondent to identify in which one of several alternative camouflaged figures a simple figure, presented as a standard, is embedded. The test is timed in two 10-min sections. Each section contains 16 disembedding tasks, the complexity of which increases as the respondent progresses through the test. The maximum score is 32 . The scores obtained by the present pool of subjects ranged from 0 to 23 . The top $8 \%(n=12)$ were selected as field-dependent (FI). Their scores ranged from 15 to 23. The bottom $8 \%(n=12)$ were classified as field-dependent 
(FD), with scores ranging from 0 to 8 . The mean for the FDs $(n=12)$ was $M=3.87(S D=2.79)$. The mean for the FIs was $M=19.62(S D=2.77)$.

\begin{abstract}
Apparatus and Signal Stimuli
The subject was seated in a small room separated from another room by a one-way mirror, which was used as a screen for backprojection of the visual stimulus. A cone constructed from translucent paper was used to restrict the subject's field of vision. The dimensions of the cone were: length $=82.5 \mathrm{~cm}$, large opening = $43 \mathrm{~cm}$, small opening $=15 \mathrm{~cm}$. The small end of the cone was placed against the mirror through which the visual stimulus was backprojected. The height of the cone was adjusted by regulation of the length of the ropes used to suspend the cone from the ceiling. The visual signal was a small red circle back-projected onto a screen from a Midway (Deluxe 300) 35-mm slide projector attenuated by a Leviton powerstat voltmeter. Noise from the projector fan or slide changes was inaudible in the experimental room. A blank slide was projected as a buffer on those trials in which the visual stimulus was not presented.
\end{abstract}

The auditory signal, transmitted to the subject via a pair of Belltone earphones, was a 250 -cps pure tone generated by a Belltone audiometer in the experimental room. Intensity was finely graded; onset and of fset were pure. Extraneous noise was blocked through the use of padded earphones and soundproofing material around the door.

\section{Imaging Tasks}

The images to be produced by the subjects corresponded to four classifications designed to tap different combinations of imagery in the auditory and visual modalities, as described below.

Visual imaging (VI). The items for the visual imaging task were "old" items previously employed by Segal and Fusella (1970). They had selected these items as representatives of the visual sense modality and on the basis of familiarity to the subjects, for example, "imagine a shoe" or "imagine an elephant."

Auditory imaging (AI). The items for this auditory imaging task were also "old" items originally selected by Segal and Fusella (1970). These items were selected as representative of the auditory sense modality, for example, "imagine the sound of a phone ringing" or "imagine the sound of a typewriter." On the basis of our pilot study, we regarded this task as one combining the effects of both auditory and visual "images."

Auditory imaging: New items (AI-N). The items employed in this condition were "new" items selected by the present investigators on the basis that they were more truly representative of the auditory sense modality than were those represented by the "old" AI items. Accordingly, the items in the AI-N list were based on onomatopoetic words requiring images of pure sounds, for example, "imagine a buzzing sound," "imagine a hissing sound," or "imagine a crackling sound."

Visual imaging: New items (VI-N). The new items employed in this task corresponded to the visual components of the old original AI tasks, for example, "imagine a telephone" or "imagine a typewriter."

\section{Procedure}

The task was individually administered in two separate sessions, each of which was $2 \mathrm{~h}$ long. Each session consisted of four phases to be described below; the exact instructions used in each phase were those reported by Segal and Fusella (1970).

Practice test trials. The practice phase was used to facilitate the subject's accommodation to the requirements of the signal detection task and to adjust the intensity level for both the visual and auditory signals. The instructions requested the subject to report whether or not a signal (sound or figure) had been presented. Upon hearing the instruction "ready," the subject was informed of the beginning of a trial. The trial ended when the subject had made one of the three responses ("sound," "picture," or "noth- ing") to the question, "What was it?" The practice period comprised 50 trials in which the subject indicated when he heard the sound or saw the figure. The signal intensity criterion, for each subject, was set at an error rate of $20 \%$ errors, following the procedure employed by Segal and Fusella (1970). Thus, there were no differences between base level performance of FD/FI groups on the practice test trials. The level obtained by this procedure for both the visual and the auditory signals was maintained throughout the remainder of the experimental session.

Preimaging test trials (signal detection without imaging). The discrimination phase was used to establish reliably the subject's sensitivity to the signals. In this phase, the subject was informed that the 60 trials were test trials. A ready signal marking the beginning of an observation trial was provided. The conclusion of a trial was marked by the subject's reply to the experimenter's question, "What was it?" The duration of each discrimination trial was $5 \mathrm{sec}$. During this period, the duration of the auditory or the visual signal, when presented, was $2 \mathrm{sec}$; the order of the three variations (auditory, visual, or no stimulus) was random.

Signal-detection-while-imaging-phase test trials. Following the discrimination phase, 120 trials of the imaging task were administered. The instructions informed the subject (1) of the nature of the experimental tasks to be presented and (2) that he would be asked to imagine various events, (3) that when he had an image he was to signal by slightly raising his hand, and (4) that shortly afterward he would be asked by the experimenter whether he thought a signal had been presented. The subject had to respond, "Yes, I heard (or saw) something," or, "No, I did not hear (or see) anything' after each trial. As in each of the other phases, the experimenter was seated behind the subject. The experimenter was "blind" as to the subject's FD or FI status.

Three different sets of 120 tasks were prepared. Each set differed from the others such that a given imagery item on one list was linked to a different signal condition (visual, auditory, or nosignal) on each of the other lists. Assignments of type of signal to a given imagined event were made by reference to a table of random numbers. A subject was administered two of these sets of tasks assigned at random with the restriction that each list would occur equally often across the FD and FI groups.

The total of 240 trials, equally divided between the two sessions, was devoted to signal detection while imaging. Each request for an image was paired with an auditory signal ( 40 trials per session), a visual signal (40 trials per session), or no signal (40 trials per session). Stated in terms of each of the four imaging tasks, there were 10 trials of imaged events paired with the auditory signal, 10 with the visual signal, and 10 with no signal within each session.

Postimaging test trials (signal detection without imaging). A second discrimination period followed the imaging task. This discrimination period differed from the first only in that $\mathbf{3 0}$ trials were given instead of 60 .

\section{Scoring and Analyses}

A response was scored 1 if the auditory or visual signal (or their absence) was correctly identified and 0 if incorrectly identified. The total scores were converted to proportions of correct responses to permit comparisons across phases. All percentages within a given phase are based on a constant denominator. For each person, there were 12 detection-of-signal-while-imaging scores (auditory and visual signal crussed by AI, VI, AI-N, and VI-N images), each of which was based on 20 observations (10 from each of the two experimental sessions).

The data were analyzed by mixed analyses of variance. Planned comparisons between means comprising significant interactions were made by Tukey's wholly significant difference (WSD) test. The rejection region for all analyses was $p<.05$. The analyses were of discrimination performance in pre- and postimaging tasks, sensitivity to detection of signal while imaging under cross- and same-modality imaging treatments, and sensitivity to detection of signal while imaging (and without imaging) by subjects differing in cognitive style. 


\section{RESULTS}

\section{Signal Detection Without Imaging}

The pre- and postimaging test trials involved only signal detection without imaging for each session. These were necessary to provide assurance that signal detection ability did not change during the course of the experimental session due to nonspecific transfer effects, such as warm-up, fatigue, boredom, adaptation, or changes in learning sets. The scores on the pretasks for both sessions were pooled for comparison with the pooled scores of the posttasks.

These data were analyzed by a mixed analysis of variance, in which the between-subjects variable was cognitive style. The within-subjects variables were pre- vs. posttest trials and type of signal. The only significant effect resulting from this analysis was that due to the type of signal, which yielded $F(2,44)=$ 7.33. This result simply indicates that the absence of a signal was detected with significantly greater accuracy $(M=.95)$ than was detection of visual $(M=.92)$ or auditory $(\mathrm{M}=.91)$ signals. This difference, although slight, may imply that a part of the processes involved in the signal detection task involves deciding, first, whether or not a signal is present and, second, if so, which signal it is. The remaining main effects and all interactions were not significant $(F<$ 1.00 ).

A further analysis was made to determine whether the detection of the absence of a signal on the nosignal trials differed significantly among the three phases (pretasks, imaging, and posttasks) for FD and FI subjects (as the between-subjects variable). None of the effects in this analysis were significant; all $F$ ratios were less than 2.95 . The FI means were .97, .94 , and .93 , respectively, for the three phases, and the FD means were $.95, .90$, and .95 , respectively, for the three phases.

These findings indicate that FI and FD subjects did not differ significantly in their ability to detect the signals or absence of the signals at the thresholds employed and that their ability to do so did not change as a result of their participation in the experimental session. Thus, whatever results are identified as a result of signal detection while imaging imply differential effects on the FD/FI groups of the task of imaging. ${ }^{~}$

\section{Cognitive Style}

Each subject's scores for the two experimental (detection of signal while imaging) sessions were pooled and analyzed by a mixed analysis of variance, in which the between-subjects variable was cognitive style and the within-subjects variables were type of signal and class of imagined event. Means involved in this analysis are summarized immediately below or in subsequent parts of this section.

This analysis yielded a significant main effect due to cognitive style [MSe $=.07821 ; \mathrm{F}(1,22)=8.64$ ], which supports the hypothesis that field-independent subjects made more correct responses over all treatments $(M=.77)$ than did field-dependent subjects $(M=.67)$. However, the qualifiedly more important result regarding performance of the two groups is the significant interaction of cognitive style with kind of signal $[F(2,44)=5.16]$. (The within MSe for signals was .0323 and the imaging MSe was .00969.) WSD tests of differences between means indicated that in detecting both the visual (VS) and auditory (AS) signals, the FI subjects $[\mathrm{M}(\mathrm{VS})=.66 ; \mathrm{M}(\mathrm{AS})=$ .71] correctly detected a greater proportion of external signals while imaging than did the FD subjects $[\mathrm{M}(\mathrm{VS})=.48 ; \mathrm{M}(\mathrm{AS})=.61)$. In each modality the difference between the performance of the two groups was significant $[t(V S)=3.25$ and $t(A S)=4.69$ ]. Under the no-signal (NS) treatment, the difference between the FI subjects $[M(N S)=.93]$ and the FD subjects $[\mathrm{M}(\mathrm{NS})=.92]$ was not significant $[\mathrm{t}(\mathrm{NS})=.84]$. These results imply that cognitive style influences detection of signals only when those signals are embedded.

\section{Accuracy of Signal Detection While Imaging}

The main analyses were conducted to provide answers to specific experimental questions, as indicated in the introduction. Since these were a priori hypotheses, raised as a result of the pilot study, specific analyses addressed to each question are statistically appropriate. Given that this study focuses on FD/FI, and given that FD vs. FI have been found to relate to many diverse phenomena, FD vs. FI differences were also explored in connection with these questions.

The first question is concerned with whether the results of the present study confirm the pilot study finding that the old AI (mixed) task with auditory signal yields a higher discrimination score than the old VI task with visual signal $[F(1,22)=7.28]$. The data related to this analysis are summarized in Table 1.

The Tukey analyses of these data indicated that, overall, the auditory signal under the old (mixed) imaging condition was detected significantly more often than was the visual signal under the old visualimaging condition. These data confirm the pilot study finding. However, the difference between the two imaging conditions is significant for the field-dependent

Table 1

Accuracy of Field-Dependent/Independent Groups in Detecting the Signal Under the "Old" Imaging Conditions

\begin{tabular}{lcccc} 
& \multicolumn{3}{c}{ Kind of Task and Signal } \\
\cline { 2 - 3 } Cognitive & \multicolumn{2}{c}{$\mathrm{AI}_{\text {AS }}$ (Mixed) } & & \multicolumn{2}{c}{ VIVS(Old) $_{\text {Style }}$} & Mean & SD & & Mean & SD \\
\cline { 2 - 5 } FD & .58 & .125 & .35 & .186 \\
FI & .64 & .219 & .55 & .230 \\
Total & .61 & .177 & .45 & .237 \\
\hline
\end{tabular}


group [M(diff $)=.23]$ but not for the field-independent group $[\mathrm{M}(\operatorname{diff})=.09]$.

The second concern was whether the two new conditions eliminated the effect described in the immediately aforegoing paragraphs. The reader will recall that auditory set of the new imaging tasks required the subject to form images of sounds uncontaminated by visual components (AI-N) and that the visual set isolated the visual elements (VI-N) from the auditory signals originally employed by Segal and Fusella $(1970,1971)$ (e.g., instead of imaging a telephone ringing, the participant was told to image a telephone). The data related to the second question were those which permitted a comparison of discrimination scores on these revised imaging tasks.

A comparison of AI-N(AS) (read as auditory images coupled with the new auditory signals) and VI-N(VS) (read as visual images coupled with the new visual signals) is displayed in Table 2.

In the analysis of these data, only the difference due to the main effect of FD/FI was significant; the mean discrimination score under imaging conditions was lower for FDs $(M=.375)$ than for FIs $(M=.61)$ $[F(1,22)=11.12]$. Pooling over both groups, the analysis showed VI-N(VS) scores to be higher than AI-N(AS) scores, but the difference is not significant. Accordingly, in answer to our question, we conclude that the new tasks did eliminate the difference between the old auditory imaging tasks with auditory signal and the old visual imaging tasks with visual signal. Since the interaction between imagery tasks and FD/FI was not significant, no additional issue is raised in terms of cognitive style.

In light of the aforegoing, the third question examines the cross-modality hypothesis by treating, in

Table 2

Accuracy of Field-Dependent/Independent Groups in Detecting the Signal With the New Imaging Tasks Under Same-Modality Conditions

\begin{tabular}{lccccc}
\hline & \multicolumn{3}{c}{ Kind of Imaging Task and Signal } \\
\cline { 2 - 3 } $\begin{array}{l}\text { Cognitive } \\
\text { Style }\end{array}$ & \multicolumn{2}{c}{ AI-N AS } & & \multicolumn{2}{c}{ VI-NVS } \\
\cline { 2 - 3 } & Mean & SD & & Mean & SD \\
\hline FD & .35 & .120 & .40 & .114 \\
FI & .61 & .139 & .62 & .218 \\
Total & .48 & .178 & .51 & .203 \\
\hline
\end{tabular}

Table 3

Accuracy in Detection of Signals Under Cross-Modality Conditions for Old and New Tasks

\begin{tabular}{|c|c|c|c|c|c|c|c|c|}
\hline \multirow{3}{*}{$\begin{array}{l}\text { Kind } \\
\text { of } \\
\text { Signal }\end{array}$} & \multicolumn{4}{|c|}{ New Task Images } & \multicolumn{4}{|c|}{ Old Task Images } \\
\hline & \multicolumn{2}{|c|}{$A I-N$} & \multicolumn{2}{|c|}{ VI-N } & \multicolumn{2}{|c|}{ AI } & \multicolumn{2}{|c|}{ VI } \\
\hline & Mean & SD & Mean & $\mathrm{SD}$ & Mean & SD & Mean & SD \\
\hline AS & .47 & .178 & .73 & .154 & .61 & .177 & .81 & .112 \\
\hline VS & .77 & .193 & .51 & .203 & .55 & .200 & .45 & .237 \\
\hline
\end{tabular}

Table 4

Accuracy of Field-Dependents/Independents in Accuracy of Detecting the Signals Under Cross-Modality Conditions on New Tasks

\begin{tabular}{|c|c|c|c|c|c|}
\hline \multirow{3}{*}{$\begin{array}{l}\text { Cognitive } \\
\text { Style }\end{array}$} & \multirow{3}{*}{$\begin{array}{c}\text { Kind of } \\
\text { Signal }\end{array}$} & \multicolumn{4}{|c|}{ Kind of Task Image } \\
\hline & & \multicolumn{2}{|c|}{ AI-N } & \multicolumn{2}{|c|}{ VI-N } \\
\hline & & Mean & $\mathrm{SD}$ & Mean & SD \\
\hline \multirow{2}{*}{ FD } & A & .35 & .120 & .70 & .102 \\
\hline & V & .71 & .212 & .40 & .114 \\
\hline \multirow[t]{2}{*}{ FI } & A & .60 & .139 & .76 & .187 \\
\hline & V & .83 & .157 & .62 & .218 \\
\hline
\end{tabular}

separate analyses, the old and new imaging tasks separately but without FD/FI as a variable. The analyses yielded a significant interaction between kind of signals and kind of images for both the new tasks, where $F(1,22)=95.93$, and the old tasks, where $F(1,22)=$ 28.05 .

The means related to these analyses are summarized in Table 3.

The results displayed in Table 3 dramatically show the cross-modality effect. All comparisons among means of discrimination scores relevant to the crossmodality effect, based on the new imaging tasks, were significant. The scores based on the old tasks show a slightly different pattern [e.g., the AL(AS) score and the AI(VS) score are higher than the VI(VS) score, but not significantly so].

These separate analyses highlight the importance of the new methodology of employing "pure" forms of the imaging tasks if the cross-modality effect is to be demonstrated.

Having demonstrated that the cross-modality effect is very strong with the new tasks, the question was asked whether the effect was equally strong for FD and FI subjects. The analysis related to this question extended the design for the immediately preceding analysis for the new tasks by incorporating FD/FI as the third variable. This analysis yielded a significant main effect due to FD/FI $[F(1,22)=13.94]$ and to the second-order interaction of FD/FI $\times$ signals $X$ images $[F(1,22)=7.18]$. No other effect was significant.

This analysis provided the basis for studying the second hypothesis; that is, FIs would be less susceptible to the processing characteristics of imaging and thus would be able to detect the externally presented signal in the same modality more accurately than would FDs. The outcome of importance in support of this hypothesis was the significant second-order interaction of FD/FI $\times$ signals $\times$ images. The means involved in this interaction are summarized in Table 4. The direction of their differences are as follows: FIs discriminated the signal under cross-modality conditions in both imaging conditions better than did FDs, but in neither comparison was the difference significant [.76 vs. .70 for VI-N(AS) and .83 vs. .71 
for AI-N(VS)]. In other words, FIs show a very small, nonsignificant superiority over FDs when image and signal are in different modalities. These data allow us to infer that both groups are equally good at imaging and/or whatever process is at work to interfere with signal detection. On the other hand, when image and signal are in the same modality, although both groups are subject to interference, the FIs achieve substantially higher scores than do the FDs. Each group's performance is significantly lower under the same modality condition than under the cross-modality condition, except for the FIs in the VI-N condition, in which the FIs' score on VI-N(AS) tasks is .76 and on the VI-N(VS) tasks is .62 . This difference is significant at $\mathrm{p}<.10>.05 .^{2}$

\section{DISCUSSION}

The results of this study indicate that processing images can interfere with the detection of a low-level signal. Mentally imaging reduces sensitivity to a threshold stimulus, with some modality specificity: that is, visual imagery reduces sensitivity to a visual stimulus more than it does to an auditory stimulus, and auditory imagery reduces sensitivity to an auditory stimulus more than it does to a visual stimulus. Fielddependent subjects were somewhat more affected by the imagery task than were field-independent subjects, and the precise nature of the imagery task (whether it was solely auditory or visual and auditory mixed) had some reasonable qualifications on the outcomes.

We speculate that this effect may be partially attributable to a division of attention, since, in the cross-modality treatments, signal detection with imaging was poorer than signal detection without imagery. Furthermore, a degree of decision-making may be involved, since absence of a signal in the signal-to-image tasks was always identified more accurately than was the presence and nature of a signal (either visual or auditory). Presumably the presence of a signal required a second decision regarding its nature. These effects were stronger for the FD subjects than for the FI subjects. The FD and FI subjects did not differ in the simple discrimination tasks or on the no-signal trials. Finally, the same-modality effect, whether with auditory or visual treatments, was stronger for FDs than for FIs. Nevertheless, signal-detection for both groups was better under cross-modality conditions than under same-modality conditions.

The results imply the possibility that the treatments differ in terms of the amount of attention demanded by the task or given by the subjects. However, if attention/distraction were the sole explanation, they should have functioned equally well, regardless of the stimulus-imagery combination. Similarly, the image should have been equally distracting for both FI and FD subjects.

A more complete explanation of the results requires the consideration that the signal was presented contiguously with the imagery event. Thus, the signal seems to have been embedded functionally into the contextual field either as an integral part of the situation (signal-to-imaging) or as imposed on the situation through the processing strategy (cognitive style) used by the subject. Each has the potential for producing a contextual field which extends some relevant characteristic(s) of the signal. As a consequence, the same-modality signal-to-imaging task results in more characteristics being shared by the imaginal and perceptual representation than in the cross-modality tasks. In other words, the image may have produced a context into which the signal was assimilated.

If context were the controlling factor in producing the cross-modality effect, then that effect should not have been obtained in this study. We assume that context would have had differential effects for FD/FIs as a direct result of camouflaging, whereby a significant figure is lost or buried by extension of its dominant lines, such that the figure becomes difficult to identify. To the extent that a figure becomes assimilated into its context, its identity is decreased correspondingly; conversely, whatever enhances sensitivity to the significant figure will reduce the effectiveness of camouflaging efforts. The critical point here is that as figure lines are extended, the extensions become a unitary part of the figure. Other kinds of adjunct stimuli would be distracting but would not necessarily result in camouflage. Successful camouflaging requires contexts that are functionally capable of assimilating the focal figure. Mere noise is insufficient. Since camouflaging was not a characteristic of our image-signal tasks, and since crossmodality effects were stronger for FDs and FIs, we are left with the conclusion that the effect is produced by the difficulty subjects had in processing two sources of inputs by the same strategy. This is another way of saying that signal detection is difficult when it occupies the same processing channels as those taken up by the image. Given a task in which the stimuli and image were intended to provide a camouflaged percept, it is expected that cross-modality differences would be enhanced, particularly for the FIs.

Although the goal of camouflaging may be accomplished externally, as it is in most deliberate camouflaging efforts, it may also be the less conscious result of imposing the products of one's own knowledge on the incoming stimuli, an assumption related to the differential ability of the FDs and FIs to disembed a camouflaged figure. The FD is assumed to be less familiar with internal cues (less differentiation) than is the FI (Witkin, Dyk, Faterson, Goodenough, 
\& Karp, 1962/1974), as assumption which accommodates the interpretation that internal cues are superimposed on external inputs to a greater degree by FDs than by FIs, such that the significant external figure may not be realized as objectively as it might otherwise be. Thus, the external stimulus, processed through low-level analyzers (Neisser, 1970), either becomes assimilated into the contextual features of the subjective image or is masked by the imaging process. The distractions between the image and signal become ambiguous when the sensory effects of the image correspond to those of the input, when the context is an extension of the figure, and when the perceptual strategies focus as much on contextual elements as on focal stimuli.

Another explanation seems plausible, in part at least, as an alternative to those proposed above, although not tested directly in this study. It has been shown (Witkin, Moore, Goodenough, \& Cox, 1977) that the social roles of FDs tend not to be as distinctly separated (compared with FIs) from the social roles of those with whom they interact. FDs tend to be particularly interested in and selectively attentive to social aspects of their environment and tend to rely more on external referents. As a consequence, they tend (more than FDs) to be attentive to and make use of prevailing social/perceptual frames of reference. This explanation becomes translated in the present experiment in terms of the degree to which images are produced by the FDs compared with FIs. Thus, in reacting to the social role aspects of the experiment, that is, responding to instructions to image, the FDs presumably would adopt this definition of the experimental requirements more stringently than would FIs. As a consequence, they would have produced "better" quality images. Although the present experiment was not designed to test this hypothesis, it nevertheless seems to be one worthy of further investigation.

In summary, this study has provided further evidence regarding the proposed competition (or commonalities) between perception and imagination as it exists in the Perky effect. Whatever the exact nature of the neurophysiological base, it may be that, as concluded by Segal and Fusella (1970), "image and signal are separate events competing for channel space; as channel space is in part modality specific, visual images and signals would use more common channel space than a visual image and an auditory signal" (p. 463). However, the distinctions between the sensitivity of the FD and FI to the signal under imaging conditions, in conjunction with the theory associated with this cognitive style, implies that a first-order or sensory isomorphism may not be all that is involved; "there need not be a concrete or first order isomorphism but rather a second order isomorphism in which the functional relations among objects as imagined must to some degree mirror the functional relations among those same objects as actually perceived"' (Shepard, 1978, p. 131).

The relation between cognitive style and the Perky effect implies that perception and imagination are susceptible to the effects of competition for channel space. At the stimulus level, they are influenced by the effects of contextual configurations which extend the structure of the stimulus. At the processing level, they share, in common, the effects on encoding of strategies which decrease or increase sensitivity to figural distinctions and may be related to hemispheric segregation of function (Zoccolotti \& Oltman, 1978). Each of these influences potentially enhances or decreases the differences between a significant figure and its contextual elements, whether such influence derives from physiological constraints or contextual definition, or is subjectively imposed.

\section{REFERENCES}

Broadbent, D. E. Perception and communication. New York: Pergamon Press, 1958.

Brooks, L. R. The suppression of visualization in reading. Quarterly Journal of Experimental Psychology, 1967, 19, 289 299.

Brooks, L. R. Spatial and verbal components of the act of recall. Canadian Journal of Psychology, 1968, 22, 349-368.

Di Vesta, F. J., Inaersoll, G. M., \& Sunshine, P. M. A factor analysis of imagery tests. Journal of Verbal Learning and Verbal Behavior, 1971, 10, 471-479.

Goldbergen, L., \& HolT, R. R. Experimental interference with reality contact: Method and group results. Journal of Nervous and Mental Disorders, 1958, 127, 99-122.

Невв, D. O. Concerning imagery. Psychological Review, 1968, 75, 466-477.

Neisser, U. Visual imagery as process and as experience. In J. S. Antrobus (Ed.), Cognition and affect. Boston: Little, Brown, 1970.

Paivio, A. Imagery and verbal processes. New York: Holt, Rinehart \& Winston, 1971.

Perky, C. W. An experimental study of imagination. American Journal of Psychology, 1910, 31, 422-452.

Richardson, A. Mental imagery. New York: Springer, 1969.

Segal, S. J. Imagery and stimulus assimilation. In P. W. Sheehan (Ed.), The function and nature of imagery. New York: Academic Press, 1972.

Segal, S. J., \& Fuselia, V. Influence of imaged pictures and sounds on detection of visual and auditory signals. Journal of Experimental Psychology, 1970, 83, 458-464.

SEgal, S. J., \& Fusella, V. Effect of images in six sense modalities on detection of visual signal from noise. Psychonomic Science, 1971, 24, 55-56.

Shepard, R. N. The mental image. American Psychologist, 1978, 33, 125-137.

Silverman, A. J., Cohen, S. I., Shmavonian, B. M., \& Greenbero, G. Psychophysical investigations in sensory deprivation: The body-field dimension. Psychosomatic Medicine, $1961,23,48-61$.

Witkin, H. A., Dyk, R. B., Faterson, H. F., Goodenough, D. R., \& Karp, S. A. Psychological differentiation. Potomac, Md: Erlbaum, 1974. (Originally published, 1962.)

Witkin, H. A., Moore, C. A., Goodenough, D. R., \& Cox, P. W. Field-dependent and field-independent cognitive styles 
and their educational implications. Review of Educational Research, 1977, 47, 1-64.

Witkin, H. A., Oltman, P. K., Raskin, E., \& KarP, A. A. A manual for the Embedded Figures Test. Palo Alto, Calif: Consulting Psychologists Press, 1971.

ZoccolotTI, P., \& Oltman, P. K. Field-dependence and lateralizations of verbal and configurational processing. Cortex, 1978, 14, 155-163.

\section{NOTES}

1. The false-alarm rates were equal in all four imaging conditions when no signal was presented. For field-independent subjects, the mean false positives were $.079, .050, .075$, and .075 for the AI-N, VI, AI, and VI-N imaging conditions, respectively; for the field-dependent subjects, the false positive rates were .079 , $.092, .104$, and .058 , respectively. None of the main effects or interactions, in an analysis of variance of these data, was significant. Accordingly, we concluded that any differences in hit rate in the main study were due to differences in sensitivity to treatments rather than to differential criteria used by subjects in following the four imaging instructions. Thus, since the subjects made high hit rates and equal false-alarm rates in the no-signal conditions, we concluded that they were using stringent criteria (not varying the criteria according to imaging instructions) in making judgments. Accordingly, it was unnecessary to employ $\mathrm{d}^{\prime}$ in the analysis.

2. As expected, parallel analyses of results obtained with the old imaging tasks were not as clear-cut. The results with visual imagery were essentially the same as those with the new imaging tasks. In the cross-modality condition, FD and FI mean scores were .80 $(\mathrm{SD}=.06)$ and $.83(\mathrm{SD}=.15)$, respectively. In the same-modality conditions, they were $.35(\mathrm{SD}=19)$ and $.55(\mathrm{SD}=.23)$, respectively. However, in the analysis of the old auditory imaging tasks, the results for the FDs were reversed compared with performance with the new tasks; in the same modality condition, their mean score was $.58(\mathrm{SD}=.12)$, and in the cross-modality condition, it was .46 $(\mathrm{SD}=.17)$. The mean scores for the FIs were equal and lower (both means were $.64, \mathrm{SD}=.20$ ) than in their performance with the new imaging tasks.

(Manuscript received November 21, 1980; revision accepted for publication October 26, 1981.) 